\title{
Prevalence of Gastrointestinal Symptoms, Severity of Dysphagia, and Their Correlation with Severity of Amyotrophic Lateral Sclerosis in a Mexican Cohort
}

\author{
Carolina Parra-Cantu ${ }^{a, b} \quad$ Arnulfo Zaldivar-Ruenes ${ }^{c} \quad$ Manuel Martinez-Vazquez $^{\mathrm{a}, \mathrm{d}}$ \\ Hector R. Martinez ${ }^{a, b}$ \\ ${ }^{a}$ Tecnologico de Monterrey, School of Medicine and Health Sciences, Monterrey, Mexico; ${ }^{b}$ ALS Clinic, Institute of \\ Neurology and Neurosurgery, Zambrano Hellion Hospital, San Pedro Garza Garcia, Mexico; 'Tecnologico de Monterrey, \\ School of Social Sciences and Government, Monterrey, Mexico; ${ }^{\mathrm{d} I n s t i t u t e}$ of Internal Medicine, Zambrano Hellion \\ Hospital, San Pedro Garza Garcia, Mexico
}

\section{Keywords}

Amyotrophic lateral sclerosis . Cohort study .

Neurodegenerative disease - Gastrointestinal symptoms .

Prevalence

\begin{abstract}
Objectives: Our study aimed to identify the prevalence and severity of gastrointestinal (GI) symptoms and dysphagia in patients with amyotrophic lateral sclerosis (ALS) and to assess whether a correlation exists between these symptoms and the severity of ALS progression. Methods: The presence and severity of Gl symptoms and dysphagia were identified by means of the Gastrointestinal Symptom Rating Scale (GSRS) and the Functional Outcome Swallowing Scale (FOSS). The Revised ALS Functional Rating Scale (ALSFRS-R) was utilized to determine the severity of ALS. Analysis of data was performed with Spearman correlations in semi-qualitative variables of clinical scales. ALSFRS-R scores were divided into 2 categories: those with mild to moderate ALS ( $\geq 40-30$ points) and patients with moderate to advanced ALS (29$\leq 20$ points). Results: We studied 43 patients with definite ALS. The most frequent GI symptoms were constipation (60.5\%), rectal tenesmus (57.5\%), hard stools (55.0\%), and borborygmus (42.5\%). The moderate to advanced ALS stage
\end{abstract}

was correlated with constipation ( $r=0.334 ; p=0.028)$, acid regurgitation $(r=0.384 ; p=0.013)$, eructation $(r=0.334 ; p=$ $0.032)$, rectal tenesmus $(r=0.498 ; p=0.001)$, and functional dysphagia $(r=0.656 ; p=<0.001)$. Conclusions: Early detection of these Gl symptoms can guide timely therapeutic decisions to avoid weight loss, a predictor for worse prognosis. This study highlights the relevance of the detection of these symptoms in ALS patients who score $\leq 29$ points in the ALSFRS-R scale to establish an appropriate treatment, prevent systemic complications, provide more comfort, and improve quality of life.

(c) 2021 S. Karger AG, Basel

\section{Introduction}

Amyotrophic lateral sclerosis (ALS) is a progressive neurodegenerative disorder characterized by the selective death of motor neurons in the cerebral cortex, brainstem, and spinal cord [1]. ALS has traditionally been considered a predominantly motor disease. The existence of additional neurological manifestations or other organ involvement suggests an alternative diagnosis $[1,2]$. However, it is increasingly recognized that nonmotor manifestations can occur. The sympathetic nervous system, parasympa-

karger@karger.com

(c) 2021 S. Karger AG, Basel

www.karger.com/ndd

Karger ${ }^{\prime \prime}=$
Correspondence to:

Hector R. Martinez, drhectormtz@yahoo.com 
Table 1. Functional Outcome Swallowing Scale

\begin{tabular}{ll}
\hline FOSS & Characteristics \\
\hline Stage 0 & Asymptomatic \\
Stage 1 & Episodic or daily symptoms of dysphagia with normal mealtime \\
Stage 2 & Dysphagia requiring significant dietary modification or prolonged mealtime \\
Stage 3 & Dysphagia with weight loss $\leq 10 \%$ of body weight with daily symptoms \\
Stage 4 & Dysphagia with weight loss $\geq 10 \%$ of body weight or severe aspiration or requiring intermittent non-oral feeding for nutrition \\
Stage 5 & Non-oral feeding for all nutrition \\
\hline
\end{tabular}

FOSS, Functional Outcome Swallowing Scale.

thetic nervous system, and enteric nervous system control the gastrointestinal (GI) system's peristalsis through neurotransmitters along with the electrical and contractile properties of smooth muscle cells $[3,4]$. Autonomic nervous system participation as well as presence of pain, urinary dysfunction, heart rate variability, and arterial hypotension further support the concept of ALS as a multisystem disease. ALS with progressive bulbar involvement may also present with prominent weakness of the muscles supplied by the glossopharyngeal and vagus nerves $[4,5]$. Therefore, dysphagia is a frequent complaint in ALS, and patients may have bronchial aspiration or respiratory muscle fatigue [6]. In addition, patients with nerve dysfunction may present with symptoms of pseudo-intestinal obstruction syndrome [7]. Furthermore, ALS-associated mutations are believed to cause defects not only in neurons, muscles, and bones but also in the intestine [8].

Despite this, there is not enough research on GI symptomatology in ALS. Identifying their prevalence allows for a more accurate assessment of ALS patients' complaints and also establishing an appropriate treatment. Hence, the aims of our study were to identify the prevalence of GI symptoms in patients with definite ALS using the Gastrointestinal Symptom Rating Scale (GSRS), along with the Functional Outcome Swallowing Scale (FOSS), and to assess whether a correlation exists between the severity of ALS determined by the Amyotrophic Lateral Sclerosis Functional Rating Scale - Revised (ALSFRS-R).

\section{Materials and Methods}

\section{Participants}

A cross-sectional design study was performed in the ALS multidisciplinary clinic at Zambrano Hellion Hospital. The Ethical Review Board of Instituto Tecnoló in Monterrey, Nuevo Leon, Mexico, approved the protocol (reference number P000435P.S.G.I.E.L.A-CEIC-CR002).

Prevalence of Gastrointestinal Symptoms in Amyotrophic Lateral Sclerosis
All procedures were conducted in accordance with the principles in the Declaration of Helsinki. All the participating patients or their guardians signed an informed consent form. The inclusion criteria were as follows: (1) patients older than 18 years with confirmed diagnosis of definite ALS according to the clinical and neurophysiological criteria of the El Escorial and Awaji-Shima; (2) cervical and cranial magnetic resonance imaging, excluding structural damage to the brain and spinal cord; (3) a functional respiratory test showing the forced vital capacity; and (4) cognitive evaluation showing ability to understand questionnaires and accept to participate in the study. The exclusion criteria included patients with (1) possible or probable ALS and (2) concomitant cardiovascular, neoplastic, infectious, or autoimmune disease.

\section{Measurement of GI Symptoms, Dysphagia, and ALS}

Progression

We identified 43 Mexican patients with definite ALS who were included in this study. Data were obtained by the medical records and patient self-reporting. The tools adopted in this study were (1) GSRS, (2) FOSS, and (3) ALSFRS-R.

The prevalence and severity of GI symptoms were assessed by the GSRS. The GSRS is a self-administered questionnaire developed to measure GI symptoms that is highly reliable and validated [9]. It is composed of a seven-point graded Likert-type scale in which 1 represents absence of symptoms and 7 represents very troublesome symptoms [10]. FOSS was utilized to assess the functional severity of dysphagia (Table 1) [11]. FOSS was calculated from detailed notes of primary care physicians and documented during evaluation at the ALS multidisciplinary clinic. ALS severity was determined by means of the ALSFRS-R score [12]. The questionnaire is structured on a 5 -point scale from 4 to 0 , where 4 represents no loss of function and 0 represents a total loss of function. In patients included in this study, ALSFRS-R scores were divided into 2 categories: those with mild to moderate ALS ( $\geq 40-30$ points) and patients with moderate to advanced ALS (29- $\leq 20$ points).

\section{Statistical Methods}

Data analyses were performed by Stata 16 software. Data entry was verified by different researchers. Patient demographic and clinical data were analyzed according to the descriptive statistics. Descriptive statistics were summarized as absolute frequencies and percentages in categorical variables and median, mean, and standard deviation in continuous variables. The relationships between the ALFRS-R score and prevalence of GI symptoms as well as dysphagia 
Table 2. Demographic and clinical characteristics of patients $(n=$ 43)

\begin{tabular}{ll}
\hline Characteristic & Patients with ALS, $n$ (\%) \\
\hline $\begin{array}{ll}\text { Gender } \\
\text { Male }\end{array}$ & $26(60.5)$ \\
$\quad$ Female & $17(39.5)$ \\
Age, years & \\
$\quad<65$ & $30(69.8)$ \\
$>65$ & $13(30.2)$ \\
ALSFRS-R by stage & \\
$\quad$ Mild to moderate $(\geq 40-30$ points $)$ & $19(44.2)$ \\
Moderate to advanced (29-S20 points) & $24(55.8)$ \\
FOSS stage & \\
0 & $7(16.3)$ \\
1 & $2(4.7)$ \\
2 & $6(13.9)$ \\
3 & $8(18.6)$ \\
4 & $11(25.6)$ \\
5 & $9(20.9)$ \\
\hline
\end{tabular}

ALS, amyotrophic lateral sclerosis; ALSFRS-R, Revised Amyotrophic Lateral Sclerosis Functional Rating Scale; FOSS, Functional Outcome Swallowing Scale.

severity were explored by correlation analyses. Analysis of data was performed with Spearman correlations in semi-qualitative variables (scales grading symptoms/presence or absence of symptom). The approach utilized for correction multiple correlations was the false discovery rate (FDR) of Benjamini and Yekutieli [13]. The correlations were grouped by the 5 dimensions assessed in the GSRS [14].

\section{Results}

We studied 43 patients with diagnosis of definite ALS. The mean age was $59.33(\mathrm{SD}=9.93)$ years. Most ALS patients were male $(60.5 \%)$ and $<65$ years $(69.8 \%)$. The average ALSFRS-R score was of $26.09(\mathrm{SD}=13.9)$ points, and the median score was $25(2-48)$ points. There were 19 patients with mild to moderate ALS ( $\geq 40-30$ points) and $24(55.8 \%)$ patients with moderate to advanced ALS (29- $\leq 20$ points). The FOSS stage among patients was from stage 0 to 5 ; there were 7 patients $(16.3 \%)$ in stage 0 , 2 patients $(4.7 \%)$ in stage 1,6 patients $(13.9 \%)$ in stage 2 , 8 patients $(18.6 \%)$ in stage 3,11 patients $(25.6 \%)$ in stage 4 , and 9 patients (20.9\%) in stage 5 (Table 2).

The most frequent GI symptoms were constipation (60.5\%), rectal tenesmus (53.5\%), hard stools (51.2\%), and borborygmus (39.5\%) (Table 3 ). The median was $3 \pm$ 2.7 symptoms for each case. The majority of them were not especially distressing for patients (the mean score was
Table 3. Frequency of Gl symptoms in 43 patients with ALS

\begin{tabular}{llll}
\hline Symptom & Frequency & Percentage & $\begin{array}{l}\text { Missing values, } \\
n(\%)\end{array}$ \\
\hline Constipation & 26 & 60.5 & 0 \\
Tenesmus & 23 & 57.5 & $3(6.97)$ \\
Hard stools & 22 & 55.0 & $3(6.97)$ \\
Borborygmus & 17 & 42.5 & $3(6.97)$ \\
Urgent need for defecation & 11 & 27.5 & $3(6.97)$ \\
Increased flatus & 11 & 27.5 & $3(6.97)$ \\
Loose stools & 10 & 25.0 & $3(6.97)$ \\
Heartburn & 10 & 25.0 & $3(6.97)$ \\
Hunger pain & 9 & 22.5 & $3(6.97)$ \\
Satiety & 9 & 22.5 & $3(6.97)$ \\
Eructation & 8 & 19.5 & $2(4.65)$ \\
Nausea & 7 & 16.3 & 0 \\
Acid regurgitation & 6 & 14.6 & $2(4.65)$ \\
Epigastric pain & 5 & 11.6 & 0 \\
Diarrhea & 2 & 4.7 & 0 \\
& & &
\end{tabular}

Gl, gastrointestinal; ALS, amyotrophic lateral sclerosis.

2 in the GSRS for minor discomfort), except constipation, hard stools, and rectal tenesmus (the mean score was 3 in the GSRS for mild discomfort). In addition, sex was associated with incontinence ( $46 \%$ women $/ 16 \%$ men; $p=$ 0.042 ), and older patients ( $>65$ years) frequently had increased flatus ( $50 \%$ vs. $17 \%$ in young; $p=0.047$ ). Furthermore, moderate to advanced ALS was correlated with constipation $(r=0.334 ; p=0.028)$, acid regurgitation $(r=$ $0.384 ; p=0.013)$, eructation $(r=0.334 ; p=0.032)$, rectal tenesmus $(r=0.498 ; p=0.001)$, and functional dysphagia $(r=0.656 ; p=<0.001)$ (Table 4).

\section{Discussion}

The prevalence of GI symptoms in patients with definite ALS has scarcely been evaluated. These GI complaints have been attributed to autonomic involvement including constipation, diffuse abdominal pain, and a feeling of fullness or nausea [15]. In our study, the most frequent GI symptoms were constipation (60.5\%), rectal tenesmus (57.5\%), hard stools (55.0\%), and borborygmus (42.5\%). Interestingly, Toepfer et al. [4] have also previously reported markedly delayed colonic transit times in 11 out of 18 studied ALS patients. Factors contributing to a delayed colonic transit in ALS may be the lack of physical activity, inadequate fiber intake due to dysphagia, and dehydration [4]. However, patients included in the study by Toepfer et al. [4] could swallow a regular solid diet and
44

Neurodegener Dis 2021;21:42-47 DOI: $10.1159 / 000517613$
Parra-Cantu/Zaldivar-Ruenes/MartinezVazquez/Martinez 
Table 4. Correlation of moderate to advanced ALSFRS-R stage and GI symptoms

\begin{tabular}{|c|c|c|c|}
\hline Variable & $\begin{array}{l}\text { Correlation with } \\
\text { moderate to advanced } \\
\text { ALSFRS-R stage }\end{array}$ & $\begin{array}{l}\text { FDR } \\
\text { (adjusted } \\
p \text { value) }\end{array}$ & $\begin{array}{l}\text { Null hypothesis } \\
\text { after } p \text { value } \\
\text { correction }\end{array}$ \\
\hline \multicolumn{4}{|l|}{ Reflux } \\
\hline Acid regurgitation & $r=0.384 ; p=0.013^{*}$ & 0.025 & Rejected \\
\hline Heartburn & $r=0.157 ; p=0.313$ & 0.050 & Accepted \\
\hline \multicolumn{4}{|l|}{ Abdominal pain } \\
\hline Epigastric pain & $r=0.176 ; p=0.257$ & 0.016 & Accepted \\
\hline Nausea & $r=0.138 ; p=0.375$ & 0.033 & Accepted \\
\hline Hunger pain & $r=-0.086 ; p=0.593$ & 0.050 & Accepted \\
\hline \multicolumn{4}{|l|}{ Indigestion } \\
\hline Eructation & $r=0.334 ; p=0.032^{*}$ & 0.012 & Accepted \\
\hline Borborygmus & $r=0.210 ; p=0.193$ & 0.025 & Accepted \\
\hline Increased flatus & $r=0.137 ; p=0.398$ & 0.037 & Accepted \\
\hline Satiety & $r=-0.002 ; p=0.986$ & 0.050 & Accepted \\
\hline \multicolumn{4}{|l|}{ Diarrhea } \\
\hline Loose stools & $r=0.202 ; p=0.210$ & 0.016 & Accepted \\
\hline Diarrhea & $r=-0.025 ; p=0.869$ & 0.033 & Accepted \\
\hline Urgent need for defecation & $r=0.025 ; p=0.877$ & 0.050 & Accepted \\
\hline \multicolumn{4}{|l|}{ Constipation } \\
\hline Tenesmus & $r=0.498 ; p=0.001^{*}$ & 0.016 & Rejected \\
\hline Constipation & $r=0.334 ; p=0.028^{*}$ & 0.033 & Rejected \\
\hline Hard stools & $r=0.145 ; p=0.369$ & 0.050 & Accepted \\
\hline \multicolumn{4}{|l|}{ Dysphagia } \\
\hline FOSS stage & $r=0.656 ; p=<0.001^{*}$ & 0.050 & Rejected \\
\hline
\end{tabular}

Correlation and significance levels are specified. Moderate to advanced ALSFRS-R stage (29- $\leq 20$ points). ALSFRS-R, Revised Amyotrophic Lateral Sclerosis Functional Rating Scale; Gl, gastrointestinal; FDR, false discovery rate; FOSS, Functional Outcome Swallowing Scale. * Significant correlations.

did not show clinical symptoms of dehydration. It has also been demonstrated that a sedentary lifestyle alone does not always necessarily influence colonic transit times significantly $[16,17]$. Therefore, one plausible explanation for our findings is that the autonomic nervous system may be affected in several ways in patients with ALS.

Additionally, several ALS patients in our cohort (25.6\%) were classified as FOSS stage 4 with a loss of $10 \%$ or more of their body weight. Hirschl et al. [18] also reported dysphagia as an important cause of malnutrition in a cohort of ALS patients. Timely detection of these GI symptoms can help guide therapeutic decisions to avoid early and marked weight loss, which has previously been shown to significantly predict a worse prognosis for ALS patients [19]. It is also important to mention that no significant correlations were found between constipation and early satiety or hunger pain in our cohort.

In our study, the moderate to advanced ALS stage was correlated with constipation $(p=0.028)$, acid regurgitation $(p=0.013)$, eructation $(p=0.032)$, rectal tenesmus $(p=0.001)$, and functional dysphagia $(p=<0.001)$. All correlations remained significant after correction with FDR-adjusted $p$ values, except for eructation. We additionally grouped patients according to sites of ALS symptom onset to evaluate if a correlation exists with GI symptoms, but no significant correlation was found in our cohort. Results can differ if patients are in hospital or at home; variations can also occur if questionnaires are answered by the patient or by their families, by mail, or by an interview with a doctor or a nurse. It is also relevant to mention that the majority of GI symptoms in our study were not especially distressing for ALS patients (the mean score was 2 in the GSRS for minor discomfort), except for constipation, hard stools, and rectal tenesmus (the mean score was 3 in the GSRS for mild discomfort).

Moreover, no correlation was found between urgent need for defecation and the severity of ALS; this observation goes in accordance with previous reports. Although pathological findings have been described in Onuf s nucleus that are consistent with denervation of the external anal sphincter, this may be insufficient to cause incontinence in longer surviving patients [20-23]. Nübling et al. [24] also found a 
high prevalence of constipation $(46 \%)$ in their cohort of ALS patients, while stool incontinence was a rare finding (9\%). However, in our cohort, sex was associated with urgent need for defection ( $46 \%$ women $/ 16 \%$ men; $p=0.042$ ). This could be explained by the fact that some women may undergo episiotomy after labor, which might predispose them to develop problems with fecal continence later in life [25]. This was not further corroborated in our cohort.

Some important correlations between the prevalence of several GI symptoms, severity of dysphagia, and severity of ALS are described (Table 4). One of the limitations of our study is that it did not consider changes in the diets of the patients included in this cohort. Moreover, results must be interpreted with caution because while the statistical significance is strong, they have low $r$ values. More studies are needed to establish the clinical importance of these associations. The correlation between GI symptoms and severity of ALS could potentially help discriminate different disease stages and improve the management of these symptoms.

\section{Conclusions}

The results in this Mexican cohort of ALS patients showed that the moderate to advanced ALS stage was correlated with constipation, acid regurgitation, eructation, rectal tenesmus, and functional dysphagia. Identification of GI symptoms is essential to give an appropriate treatment and provide more comfort for the final phases of life.

\section{Acknowledgements}

The authors thank the participants and their families for their invaluable contributions to research.

\section{Statement of Ethics}

Published research complies with the guidelines for human studies and was conducted ethically in accordance with the World Medical Association Declaration of Helsinki. The study protocol was approved by the Ethical Review Board of Tecnologico de Monterrey, School of Medicine and Health Sciences. The protocol reference number is P000435-P.S.G.I.E.L.A-CEIC-CR002. A written informed consent was obtained from all individual participants included in this study.

\section{Conflict of Interest Statement}

The authors have no conflicts of interest to declare.

\section{Funding Sources}

The study was supported by the Institute of Neurology and Neurosurgery at Zambrano Hellion Hospital.

\section{Author Contributions}

C.P.-C. and M.M.-V. conceived the presented idea. C.P.-C. implemented the research and wrote the manuscript. A.Z.-R. performed the calculations and analysis of the results. C.P.-C., M.M.V., and H.R.M. contributed to the interpretation of results. H.R.M. was in charge of overall direction and planning. All authors provided critical feedback, discussed results, and commented on the manuscript.

\section{Data Availability Statement}

The data that support the findings of this study are openly available in Figshare at https://doi.org/10.6084/m9.figshare. 14707329.v1 [26]. Further inquiries can be directed to the corresponding author.

\section{References}

1 Brooks BR, Miller RG, Swash M; World Federation of Neurology Research Group on Motor Neuron Diseases. El Escorial revisited: revised criteria for the diagnosis of amyotrophic lateral sclerosis. Amyotroph Lateral Scler Other Motor Neuron Disord. 2000;1(5):2939.

2 Ravits JM, La Spada AR. ALS motor phenotype heterogeneity, focality, and spread: deconstructing motor neuron degeneration. Neurology. 2009;73(10):805-11.

3 de Carvalho M, Pinto S. Is amyotrophic lateral sclerosis (ALS) a multi system disorder? Reply. Muscle Nerve. 2013;47(4):620-1.

4 Toepfer CF, Klauser A, Riepl RL, Muller-Felber W, Pongratz D. Gastrointestinal dysfunction in amyotrophic lateral sclerosis. Amyo- troph Lateral Scler Other Motor Neuron Disord. 2000;1(1):15-9.

5 Martínez H, Escamilla-Ocañas C, Hernández-Torre MJN. Síntomas neurológicos extra-motores en pacientes con esclerosis lateral amiotrófica. Neurología. 2018;33(7):474-6.

6 Cykowski MD, Takei H, Schulz PE, Appel SH, Powell SZ. TDP-43 pathology in the basal forebrain and hypothalamus of patients with amyotrophic lateral sclerosis. Acta Neuropathol Commun. 2014;2(1):1-11.

7 Camilleri M, Balm RK, Low PA. Autonomic dysfunction in patients with chronic intestinal pseudo-obstruction. Clin Auton Res. 1993;3(2):95-100.

8 Zhang Y-G, Wu S, Yi J, Xia Y, Jin D, Zhou J, et al. Target intestinal microbiota to alleviate disease progression in amyotrophic lateral sclerosis. Clin Ther. 2017;39(2):322-36.

9 Kulich KR, Madisch A, Pacini F, Piqué JM, Regula J, Van Rensburg CJ, et al. Reliability and validity of the Gastrointestinal Symptom Rating Scale (GSRS) and Quality of Life in Reflux and Dyspepsia (QOLRAD) questionnaire in dyspepsia: a six-country study. Health Qual Life Outcomes. 2008;6(1):12.

10 Svedlund J, Sjödin I, Dotevall GJ. GSRS: a clinical rating scale for gastrointestinal symptoms in patients with irritable bowel syndrome and peptic ulcer disease. Dig Dis Sci. 1988;33(2):129-34.

11 Salassa JR. A functional outcome swallowing scale for staging oropharyngeal dysphagia. Dig Dis. 1999;17(4):230-4. 
12 Cedarbaum JM, Stambler N, Malta E, Fuller C, Hilt D, Thurmond B, et al. The ALSFRS-R: a revised ALS functional rating scale that incorporates assessments of respiratory function. BDNF ALS Study Group (Phase III). J Neurol Sci. 1999;169(1-2):13-21.

13 White T, van der Ende J, Nichols TE. Beyond Bonferroni revisited: concerns over inflated false positive research findings in the fields of conservation genetics, biology, and medicine. Conserv Genet. 2019;20(4):927-37.

14 Kamiya T, Shikano M, Kubota E, Mizoshita T, Wada T, Tanida S, et al. A multicenter randomized trial comparing rabeprazole and itopride in patients with functional dyspepsia in Japan: the NAGOYA study. J Clin Biochem Nutr. 2017;60(2):130-5.

15 Shindo K, Shimokawa C, Watanabe H, Iida H, Ohashi K, Nitta K, et al. Chronological changes of sympathetic outflow to muscles in amyotrophic lateral sclerosis. J Neurol Sci. 2004; 227(1):79-84.

16 Robertson G, Meshkinpour H, Vandenberg K, James N, Cohen A, Wilson A. Effects of exercise on total and segmental colon transit. J Clin Gastroenterol. 1993;16(4):300-3.
17 Sesboüé B, Arhan P, Devroede G, LecointeBesançon I, Congard P, Bouchoucha M, et al. Colonic transit in soccer players. J Clin Gastroenterol. 1995;20(3):211-4.

18 Hirschl DA, Park JH, Heiman-Patterson TD, Ovsiowitz M, Siva B, Paylor TA, et al. The prevalence of gastrointestinal symptoms in patients with amyotrophic lateral sclerosis. Am J Gastroenterol Suppl. 2002;97(9):S283.

19 Limousin N, Blasco H, Corcia P, Gordon PH, De Toffol B, Andres C, et al. Malnutrition at the time of diagnosis is associated with a shorter disease duration in ALS. J Neurol Sci. 2010;297(1-2):36-9.

20 Kihira T, Yoshida S, Yoshimasu F, Wakayama I, Yase Y. Involvement of Onuf's nucleus in amyotrophic lateral sclerosis. J Neurol Sci. 1997;147(1):81-8.

21 Carvalho M, Schwartz MS, Swash M. Involvement of the external anal sphincter in amyotrophic lateral sclerosis. Muscle Nerve. 1995; 18(8):848-53.

22 Mannen T, Iwata M, Toyokura Y, Nagashima K. Preservation of a certain motoneurone group of the sacral cord in amyotrophic lateral sclerosis: its clinical significance. J Neurol Neurosurg Psychiatry. 1977;40(5):464-9.
23 Baltadzhieva R, Gurevich T, Korczyn ADJ. Autonomic impairment in amyotrophic lateral sclerosis. Coin. 2005;18(5):487-93.

24 Nübling GS, Mie E, Bauer RM, Hensler M, Lorenzl S, Hapfelmeier A, et al. Increased prevalence of bladder and intestinal dysfunction in amyotrophic lateral sclerosis. Amyotroph Lateral Scler Frontotemporal Degener. 2014;15(3-4):174-9.

25 Rusavy Z, Karbanova J, Jansova M, Kalis V. Anal incontinence and fecal urgency following vaginal delivery with episiotomy among primiparous patients. Int J Gynaecol Obstet. 2016;135(3):290-4.

26 Parra-Cantu C. Database: Prevalence of gastrointestinal symptoms, severity of dysphagia, and their correlation with severity of amyotrophic lateral sclerosis in a Mexican cohort [Internet]. figshare. 2021 [cited 2021 Jun 1]. Available from: https://figshare.com/articles/ dataset/Database_Prevalence_of_gastrointestinal_symptoms_severity_of_dysphagia and_their_correlation_with_severity_of_ amyotrophic_lateral_sclerosis_in_a_Mexican_cohort/14707329/1. 